\title{
Papel del endotelio en hipertensión inducida por el embarazo: ¿alteraciones comunes a las de la aterosclerosis?
}

\author{
The role of endothelium in pregnancy induced hypertension: alterations common with
} those of atherosclerosis?

\author{
Patricio López-Jaramillo ${ }^{1,2}$, Katherine Sotomayor-Rubio', \\ Arístides Sotomayor-Rubio ${ }^{1}$, Cristina López-López ${ }^{3}$ \\ ${ }^{1}$ Dirección de Investigaciones, Fundación Oftalmológica de Santander (FOSCAL), Floridablanca, Santander, Colombia. \\ ${ }^{2}$ Instituto Masira, Facultad de Medicina, Universidad de Santander (UDES), Bucaramanga, Santander, Colombia. \\ ${ }^{3}$ Facultad de Medicina, Universidad Autónoma de Bucaramanga (UNAB), Santander, Colombia.
}

\begin{abstract}
Resumen
La hipertensión inducida por el embarazo, cuya forma proteinúrica es denominada preeclampsia (PE), es una alteración que ocurre en el segundo trimestre del embarazo, y se caracteriza por la presencia de hipertensión y proteinuria. Durante el embarazo normal ocurren cambios fisiológicos adaptativos que incluyen insulino-resistencia (IR), hiperlipidemia, hipercoagulabilidad, inflamación y un estado circulatorio hiperdinámico. Estos cambios se expresan de una forma exagerada en las mujeres que desarrollan PE, alteraciones que están presentes también en el clúster de factores de riesgo que conforman el denominado síndrome metabólico (SM), el cual es un factor de riesgo para el desarrollo de diabetes mellitus tipo 2 (DM2) y enfermedad cardiovascular (ECV). En la presente revisión proponemos que la disfunción endotelial es la alteración común que explica la presencia de estas dos enfermedades comunes en América Latina.

Palabras clave: Endotelio vascular, embarazo, hipertensión, síndrome metabólico, preeclampsia, aterosclerosis, enfermedad cardiovascular.

Abstract

Pregnancy induced hypertension, called preeclampsia (PE) when accompanied by proteinuria, is an alteration that occurs in the second trimester of pregnancy and is characterized by presence of hypertension and proteinuria. Adaptative physiologic changes occur during normal pregnancy including insulin-resistance (IR), hyperlipidemia, hypercoagulability, inflammation, and hyperdynamic circulatory status. Expression of these changes is exaggerated in women developing PE. These alterations are also present in the metabolic syndrome (MS) risk factors cluster, which is in turn a risk factor for developing type 2 diabetes mellitus (DM2) and cardiovascular disease (CVD). In this review endothelial dysfunction is proposed as a common alteration that explains the presence of these two common diseases in Latin America.
\end{abstract}

Keywords: Vascular endothelium, pregnancy, hypertension, metabolic syndrome, preeclampsia, atherosclerosis, cardiovascular disease.

An Fac med. 2014;75(4):361-6 / doi: http://dx.doi.org/10.15381/anales.v75i4.10858

\section{INTRODUCCIÓN}

La preeclampsia es la forma proteinúrica de la hipertensión inducida por el embarazo (HIE) y en Colombia es la principal causa de mortalidad materna, ocasionando el $42 \%$ de los óbitos maternos. Se asocia con un aumento de 5 veces en la mortalidad perinatal, explicando parcialmente el por qué la mortalidad materna en países como Colombia es 10 veces más frecuente que en los Estados Unidos ${ }^{(1)}$. A pesar de su importancia en términos de Salud Pública, su etiopatogenia no ha sido aclarada totalmente. Nosotros hemos postulado que es una enfermedad compleja multicausal para cuyo desarrollo participan una serie de factores nutricionales, medioambientales y genéticos que tienen como vía final común una alteración en la función del endotelio vascular, caracterizada por disminución en la producción de sustancias vasodilatadoras como el óxido nítrico (NO) y la prostaciclina y por un exceso en la liberación de sustancias vasoconstrictoras y prooxidantes, como la endotelina y los radicales libres de oxígeno ${ }^{(2-4)}$.

Por otro lado, la enfermedad cardiovascular aterosclerótica (ECVA) es la causa principal de muerte globalmente. Así, se ha proyectado que para el año 2020 cerca de 20 millones de personas morirán por esta causa. Actualmente, aproximadamente 17,5 millones de personas fallecen anualmente por ECVA, lo que representa aproximadamente un 30\% de todas las muertes. De estas muertes, 7,6 millones son debidas a infarto agudo de miocardio (IAM) y 5,7 millones a accidente cerebrovascular (ACV) ${ }^{(5)}$.

La ECVA es considerada como un proceso inflamatorio en el que varios mecanismos interactúan con factores 
de riesgo para iniciar, propagar y activar la alteración en la función del endotelio vascular ${ }^{(6,7)}$. La ECVA en las mujeres es actualmente de las principales causas de morbilidad y mortalidad a nivel mundial. Una historia de PE está asociada años más tarde con un aumento del riesgo de ECVA, pero no de otras enfermedades crónicas, como cáncer ${ }^{(8-14)}$, observación que ha llevado a proponer que la $\mathrm{PE}$ es una manifestación temprana y transitoria de disfunción endotelial que con los años dará lugar a ECVA, la cual es una enfermedad crónicamente progresiva y en la mayoría de los casos irreversible.

\section{ADAPTACIONES HEMODINÁMICAS DURANTE EL EMBARAZO NORMAL Y SUS ALTERACIONES EN LA PREECLAMPSIA}

Durante el embarazo, las adaptaciones hemodinámicas que se observan normalmente son aumento del volumen sanguíneo, aumento del gasto cardiaco y disminuida resistencia vascular periférica. La producción endotelial de prostaciclina fue postulada como el mecanismo que interviene de la vasodilatación fisiológica del embarazo y que su menor producción se asocia a PE ${ }^{(3)}$. Estudios posteriores demostraron que, si bien la síntesis vascular de prostaciclina está disminuida en la mujer con $\mathrm{PE}$, en la mujer embarazada normal la producción de prostaciclina es similar a la de la mujer no embarazada, por lo que se planteó que la prostaciclina tiene un papel en las manifestaciones clínicas de la enfermedad. Sin embargo, probablemente no es la sustancia que primariamente está involucrada en la vasodilatación que caracteriza y explica las adaptaciones hemodinámicas del embarazo normal. Por ello propusimos que debía ser el NO la sustancia que participe de la vasodilatación que caracteriza al embarazo normal y demostramos que en las embarazadas normales la producción de $\mathrm{NO}$, valorada por los niveles plasmáticos de nitritos, está aumentada en relación a controles no embarazadas; así como también está incrementada la actividad del $\mathrm{NO}$ evaluada por las concentraciones de monosfato cíclico de guanosina (GMPc), el efector de las acciones vasculares del $\mathrm{NO}$, tanto en plasma con en orina, y que el aumento en la producción endotelial de NO durante la gestación está relacionada con un aumento de la actividad de la NOSe, estimulada por un mayor estrés de fricción o por el incremento fisiológico de estrógenos durante la gestación ${ }^{(15-24)}$.

Si bien los resultados que demuestran una mayor producción y bioactividad del NO durante la gestación son consistentes entre los diferentes grupos que han trabajado en el tema, no ocurre lo mismo durante la PE. Los resultados son contradictorios con grupos reportando niveles plasmáticos de nitritos y nitratos disminuidos, iguales o aumentados, en relación a las embarazadas normales. Estas discrepancias pueden deberse a que la cuantificación de nitritos y nitratos, para evaluar la producción de NO, está influenciada por una serie de factores como dieta, medicamentos y por su excreción urinaria. Sin embargo, cuando se corrige los valores de nitritos y nitratos por estos factores, los resultados reportados continúan siendo contradictorios ${ }^{(3)}$.

\section{FACTORES QUE AFECTAN LA PRODUCCIÓN DE ÓXIDO NÍTRICO Y SUPERÓXIDO EN LA PREECLAMPSIA}

La adecuada disponibilidad del cofactor tetrahidrobiopterina $\left(\mathrm{BH}_{4}\right)$ es fundamental para que la NOSe reciba del fosfato de dinucleótido de nicotinamida y adenina reducida (NADPH) y almacene luego suficiente cantidad de electrones para transformar oxígeno y $\mathrm{L}$-arginina en $\mathrm{NO}$ y L-citrulina. Es conocido que, en condiciones de una menor disponibilidad de $\mathrm{BH}_{4}$, la $\mathrm{NOS}$ no es capaz de catalizar la oxidación con cinco electrones que lleva a la transformación de L-arginina en $\mathrm{NO}$, pero sí es capaz de donar un electrón para el oxígeno y dar lugar a la formación de radical libre de oxígeno $(\mathrm{RO})$ en una reacción con un solo electrón. Por tanto, en condiciones de deficiencia de $\mathrm{BH}_{4}$, la NOSe deja de producir $\mathrm{NO}$ y produce más $\mathrm{RO}{ }^{(25-30)}$.

Una concentración adecuada de Larginina también es necesaria para prevenir la formación de RO por acción de la NOSe. Así, se ha demostrado que la producción de $\mathrm{RO}$ aumenta en la medida que las concentraciones intracelulares de L-arginina disminuyen y que la adición del aminoácido disminuye la producción de $\mathrm{RO}$. La deficiencia de $\mathrm{BH} 4$, más que la de L-arginina, es la que mayormente induce a que la NOS se convierta en una enzima productora de RO antes que de NO. El RO es barrido por acción de la enzima superóxido dismutasa (SOD), que la transforma en $\mathrm{H}_{2} \mathrm{O}_{2}$, la que es luego metabolizada por acción de una catalasa. El $\mathrm{RO}$ reacciona con el $\mathrm{NO}$, tres veces más rápidamente que con la SOD, para formar el radical libre peroxinitrito (ONOO), el cual es un poderoso oxidante. Normalmente, la cantidad de ONOO que se forma es pequeña, y depende únicamente de la concentración de RO. Si esta se eleva, aumenta la formación de ONOO, y por esta vía disminuye la biodisponibilidad de $\mathrm{NO}^{(31-35)}$.

La producción de $\mathrm{NO}$ se afecta también por la acumulación de dimetil arginina asimétrica (ADMA), un inhibidor endógeno de la síntesis de $\mathrm{NO}$, la cual es metabolizada por la acción de la enzima dimetilarginina diaminohidrolasa, la cual está colocalizada en células que contienen NOS. Se ha demostrado que las enfermedades que cursan con alteraciones en la bioactividad de $\mathrm{NO}$ cursan también con concentraciones aumentadas de ADMA; tal es el caso de hipercolesterolemia, falla cardiaca congestiva, aterosclerosis, insuficiencia renal, claudicación intermitente, microangiopatía trombótica ${ }^{(33-36)}$. En la preeclampsia, algunos grupos demostraron un aumento en las concentraciones circulantes de ADMA. Sin embargo, en nuestras pacientes no encontramos diferencias en los niveles de ADMA entre mujeres con PE y embarazadas normales ${ }^{(37,38)}$. 
Los radicales libres de oxígeno son producidos continuamente, especialmente como resultado de la actividad de la enzima NADPH oxidasa. También se produce $\mathrm{RO}$ en el endotelio durante el metabolismo del acido araquidónico, por acción de las prostaglandino sintetasas, lipoxigenasa y citocromo P450 y por acción de factores circulantes como LDL, TNFa y xantina oxidasa ${ }^{(39,40)}$.

\section{DESBALANCE ENTRE NO/ O2 EN PREECLAMPSIA Y ATEROSCLEROSIS}

A pesar de los resultados paradójicos en la medición de $\mathrm{NO}$ a través de la cuantificación de niveles séricos y plasmáticos de $\mathrm{NO}_{2} / \mathrm{NO}_{3}$, las concentraciones de cGMP sí se mostraron disminuidas en mujeres preeclámpticas, tanto en plasma como orina, dando a entender que existe una biodisponibilidad disminuida de NO. Lo mismo ocurre en la aterosclerosis, donde la disminución en la biodisponibilidad de $\mathrm{NO}$ es observada incluso antes de que los cambios vasculares estructurales ocurran, explicando también la similitud de las manifestaciones clínicas de ambas enfermedades, preeclampsia y aterosclerosis, que incluyen vasoconstricción y disfunción endotelial como las convergentes más importantes ${ }^{(3)}$.

Dentro de los mecanismos que conllevan a la disminución de la biodisponibilidad de $\mathrm{NO}$ que comparten ambas enfermedades, se ha descrito:

a. Presencia de alteraciones polimórficas de la NOSe que resulta en una baja actividad enzimática ${ }^{(23)}$.

b. Mecanismos inhibidores endógenos de NOSe, como la acumulación de dimetilarginina asimétrica (ADMA), han sido involucrados en aterosclerosis y $\mathrm{PE}{ }^{(36,37)}$.

c. La deficiencia de cofactores necesarios para la actividad normal de la eNOS, como iones de $\mathrm{Ca}$ y $\mathrm{BH} 4$, ha sido descrita en la preeclampsia y demostrada en la aterosclerosis por estudios clínicos, en donde la administración de BH4 mejoró la disfunción endotelial asociada con hipercolesterolemia, aterosclerosis, HTA y tabaquismo. Además, la incubación de vasos sanguíneos con sepiapterin, un precursor de $\mathrm{BH} 4$, mejoró la producción de los factores relajantes derivados del endotelio y disminuyó la producción de superóxidos ${ }^{(25-30)}$.

d. El aumento del estrés oxidativo ha sido observado en ambas enfermedades ${ }^{(39,40)}$. En los vasos ateroscleróticos, por ejemplo, se ha encontrado un incremento en una variedad de enzimas que producen superóxido, como la oxidasa, cicloxigenasa y NADPH oxidasa, siendo esta última la que tiene el papel más importante en las células vasculares ${ }^{(39)}$.

\section{ESTRÉS OXIDATIVO EN PREECLAMPSIA Y ATEROSCLEROSIS}

El estrés oxidativo ha sido postulado como el precursor del daño endotelial en la aterosclerosis, debido a la oxidación de LDL y su acumulación en el espacio subendotelial. En pacientes con preeclampsia también se ha demostrado la presencia de estrés oxidativo y en estas mujeres existe una reducción de la respuesta antioxidante, de los niveles de nutrientes antioxidantes y un aumento de la peroxidación lipídica ${ }^{(41-43)}$. La alteración de la función endotelial debida a las especies reactivas del oxígeno generadas en el espacio intervelloso, se puede vincular con la reducción de perfusión placentaria y enfermedad sistémica materna en la PE. La invasión incompleta del trofoblasto conduce al fracaso de la conversión de las paredes gruesas y tortuosas de las arterias espirales a vasos sinusoidales con paredes flácidas y de baja resistencia. La invasión incompleta resulta en problemas con la perfusión placentaria. Este daño que produce hipoxia / reperfusión da lugar a una mayor expresión de la xantina oxidasa y NADPH oxidasa y el consiguiente aumento de la generación de anión superóxido. El aumento en la generación de prooxidantes inclina la balanza a favor del estrés oxidativo, lo que lleva a un aumento de la peroxidación lipídica. Las especies reactivas del oxígeno, en general, son muy lábiles. Sin embargo, existen varias posibilidades de transferencia de sus efectos al endotelio. La más simple sería la injuria oxidativa debida a los productos de la oxidación lipídica, como malondialdehido, el cual se encuentra elevado en el plasma de las mujeres preeclámpticas. Por otra parte, los neutrófilos y monocitos que pasan por el espacio intervelloso se activan por la exposición de las especies reactivas del oxígeno e interactúan con las células endoteliales, generando estrés oxidativo ${ }^{(39-47)}$.

Las mujeres con una dieta deficiente en nutrientes antioxidantes estarían en un mayor riesgo de desarrollar PE. Los antioxidantes como la vitamina $\mathrm{C}$ y la vitamina $E$ han sido estudiados en algunos ensayos para la prevención de la preeclampsia. La intervención temprana con la administración de suplementos de vitamina $\mathrm{E}$ y $\mathrm{C}$, en mujeres entre las semanas 16 a 22 de embarazo resultó en una reducción significativa de PE en el grupo suplementado. La suplementación en mujeres con preeclampsia establecida no dio lugar a ningún beneficio ${ }^{(48,49)}$.

\section{SÍNDROME METABÓLICO EN ATEROSCLEROSIS Y PREECLAMPSIA}

El estudio INTERHEART ${ }^{(50)}$ mostró que un aumento en la proporción de apo B / apo A1, tabaquismo, diabetes, hipertensión, obesidad abdominal, estrés psicosocial fueron asociados positivamente con la presencia de un IAM, mientras que el consumo adecuado de frutas y hortalizas, el consumo moderado de alcohol, y el ejercicio se asociaron con un menor un menor riesgo de tener este tipo de evento. Las dietas hipercalóricas, con alto contenido en grasa que son bajas en fibra, se han convertido en alimentos comunes en los países en vía de desarrollo, que junto a la creciente falta de actividad física que ha acompa- 
ñado a la urbanización, han contribuido a producir aumento en la prevalencia de obesidad, especialmente abdominal. La obesidad abdominal junto con otros dos factores de riesgo constituye el síndrome metabólico (SM) ${ }^{(51-57)}$.

En condiciones normales de embarazo, las mujeres tienen algún grado de insulino-resistencia (IR), especialmente en el tercer trimestre de gestación. El aumento de IR determinado temprano en el embarazo por el índice homeostatic model assessment (HOMA) se asocia con el desarrollo posterior de HIE, por lo que se ha propuesto que el índice HOMA es un método útil para evaluar las mujeres en situación de riesgo para el desarrollo de PE. El SM se ha relacionado con disfunción endotelial, mecanismo que podría explicar los altos valores de la PA encontrados en las mujeres con mayor HOMA. Un IMC superior a $30 \mathrm{~kg} / \mathrm{m} 2$ y un aumento de peso sustancial durante el embarazo se han asociado con mayor riesgo de desarrollar PE y una correlación positiva entre IMC y niveles de HOMA. Además, es bien conocido que el aumento de adiposidad abdominal está estrechamente relacionado con el aumento de IR, de las concentraciones de insulina y de disfunción endotelial, ya que el tejido graso visceral produce sustancias proinflamatorias que intervienen en la disfunción endotelial ${ }^{(58-64)}$.

\section{INFLAMACIÓN EN ATEROSCLEROSIS Y PREECLAMPSIA}

La inflamación es uno de los mecanismos fisiopatológicos comunes que comparten la PE y la aterosclerosis. En la aterosclerosis existe acumulación de células mononucleares, migración y proliferación de músculo liso y formación de tejido fibroso que conducen a la formación de la placa aterosclerótica. En ambas entidades, marcadores plasmáticos de inflamación como proteína $\mathrm{C}$ reactiva (PCR), interleuquina- 6 (IL-6), TNF- $\alpha$, están aumentados. La PCR ha sido propuesta como un factor de riesgo independiente para la en- fermedad coronaria. En embarazadas en las primeras semanas de gestación, la vasodilatación mediada por flujo (FMD) esta disminuida y asociada a la inflamación, antes que se presenten las manifestaciones clínicas de la PE ${ }^{(65-70)}$.

\section{PAPEL DE LA INFECCIÓN EN ATEROSCLEROSIS Y PREECLAMPSIA}

La infección ha sido propuesta como una de las causas de aterosclerosis y PE. Títulos elevados de anticuerpos contra Chlamydia han sido encontrados en pacientes con enfermedad arterial coronaria (EAC) y se sugirió que esta bacteria puede ayudar a desarrollar aterosclerosis. Otros gérmenes que también pueden contribuir a EAC son los de la familia del herpes virus. El citomegalovirus se encuentra en las lesiones ateroscleróticas y puede modular la respuesta inmune celular, así como la actividad de células vasculares e incrementar la aterosclerosis. En mujeres con PE se ha observado un mayor seroprevalencia de IgG para C. pneumoniae y mayor incidencia de infecciones de vías urinarias, vaginales y periodontales, asociada a mayores concentraciones de PCR. Las mujeres con estas infecciones, al ser sometidas a terapia con antibiótico, presentan una disminución de la incidencia de PE (71-77).

\section{EVOLUCIÓN DE PREECLAMPSIA A ATEROSCLEROSIS}

Varios estudios han demostrado que existe una relación entre PE y riesgo de ECV a futuro. Un metaanálisis, que incluyó estudios de cohorte retrospectivos y prospectivos en los cuales se determinó el riesgo a futuro de enfermedad cardiovascular (IAM, HTA, ACV, tromboembolismo venoso), cáncer y mortalidad total en mujeres que presentaron PE, demostró un incremento en el riesgo de ECV en mujeres que tuvieron PE en relación las mujeres con embarazo normal. Actualmente, se considera que una historia de PE es un factor de riesgo cardiovascular en mujeres y que dicha asociación podría reflejar una causa común para $\mathrm{PE}$ y ECV, o a la existencia de un efecto de PE sobre el desarrollo de la enfermedad O ambas ${ }^{(78)}$.

La ECV va precedida de lesiones ateroscleróticas y disfunción endotelial asociadas a factores de riesgo que son bien compartidos entre ECV y PE, incluyendo HTA preexistente, trombofilia (mutaciones del factor II o factor $\mathrm{V}$ de Leiden), dislipidemia, obesidad, insulinorresistencia, DM2, inflamación e historia familiar de ECV o ACV. Las estrías grasas que preceden al ateroma prevalecen en gente joven, nunca causan síntomas y pueden progresar a ateroma o eventualmente desaparecer. Pero, en personas con hipercolesterolemia o inflamación generalmente presentes en mujeres con historia de PE causan activación focal del endotelio en arterias de pequeño y mediano tamaño. Los excesos de LDL infiltran la arteria y causan una respuesta inflamatoria en la pared de la arteria y las modificaciones enzimáticas y oxidativas producen liberación de lípidos inflamatorios que inducen a las células endoteliales a que expresen moléculas de adhesión que atraen plaquetas, macrófagos, células T, citoquinas y más células inmunes inflamatorias que rodean el centro de la placa, causando su activación, transformando la placa estable en una estructura vulnerable e inestable que se puede romper, inducir trombosis y producir un síndrome coronario agudo.

Debido a que muchos marcadores de disfunción endotelial están presentes en mujeres con PE, incluso antes del comienzo de las manifestaciones clínicas, esta disfunción endotelial puede servir como factor causal y no solo ser un resultado de la HIE. Es por esta razón que proponemos que la disfunción endotelial es una causa común de ambas enfermedades, ya que las mujeres que presentan factores de riesgo para aterosclerosis son más susceptibles a desarrollar preeclampsia y esta última acelera el proceso a aterosclerosis, por exacerbar el daño endotelial ya presente ${ }^{(79-83)}$. 


\section{REFERENCIAS BIBLIOGRÁFICAS}

1. Lopez-Mayorga A, López-Jaramillo P. Epidemiología de la hipertensión inducida por el embarazo. En: López-Jaramillo P (ed), Hipertensión Inducida por el Embarazo. Fisiopatología y Prevención. Quito: UNICEF, Ediciones Cientificas. 1993:1-13

2. Reyes LM, Garcia RG, Ruiz SL, Camacho PA, Ospina MB, Aroca G, Accini JL, Lopez-Jaramillo P. Risk factors for preeclampsia in women from Colombia: A case-control study. Plos One. 2012;7(7):e41622. doi:10.1371/journal.pone.0041622.

3. Lopez-Jaramillo P, Arenas WD, Garcia RG, Rincon MY, Lopez M. The role of the L-argininenitric oxide pathway in preeclampsia. Ther Adv Cardiovasc Dis. 2008 Aug;2(4):261-75. doi: $10.1177 / 1753944708092277$

4. Lopez-Jaramillo P, Garcia RG, Reyes L, Ruiz S. Appropriated prenatal care: the best way to prevent preeclampsia in Andean Countries. Colombia Medica. 2009;40;226-30.

5. Murray CJ, Lopez AD. Global mortality, disability, and the contribution of risk factors: Global Burden of Disease Study. Lancet. 1997;349:1436.

6. Hansson GK. Inflammation, atherosclerosis, and coronary artery disease. N Engl J Med. 2005;352:1685-95.

7. Lopez-Jaramillo P, Lahera V, Lopez-Lopez J. Epidemic of cardiometabolic diseases: A Latin American point of view. Ther Adv Cardiovasc Dis. 2011 Apr;5(2):119-31. doi: 10.1177/1753944711403189

8. Jacobs AK, Eckel RH. Evaluating and managing cardiovascular disease in women: understanding a woman's heart. Circulation. 2005;111:383-4.

9. Smith GC, Pell JP, Walsh D. Pregnancy complications and maternal risk of ischaemic heart disease: a retrospective cohort study of 129,290 births. Lancet. 2001;357:2002-6.

10. Sattar N, Greer IA. Pregnancy complications and maternal cardiovascular risk: opportunities for intervention and screening? BMJ. 2002;325:157-60.

11. Haukkamaa L, Salminen M, Laivuori H, Leinonen $\mathrm{H}$, Hiilesmaa ¡V, Kaaja R. Risk for subsequent coronary artery disease after preeclampsia. Am J Cardiol. 2004;93:805-8.

12. Funai EF, Friedlander $Y$, Paltiel O, Tiram E, Xue X, Deutsch $L$, et al. Long-term mortality after preeclampsia. Epidemiol. 2005;16:206-15.

13. Wilson BJ, Watson MS, Prescott GJ, Sunderland S, Campbell DM, Hannaford P, et al. Hypertensive diseases of pregnancy and risk of hypertension and stroke in later life: results from cohort study. BMJ. 2003;326:845

14. Hannaford P, Ferry S, Hirsch S. Cardiovascular sequelae of toxaemia of pregnancy. Heart. 1997;77:154-8.

15. Félix C, López A, Delgado F, Amores E, Narváez M, López-Jaramillo P: Vascular production of prostacyclin in Andean women with pregnancyinduced hypertension. Braz J Med Bio Res. 1991;24:59-62.

16. Félix $C$, Jácome $P$, López $A$, Moya $W$, Narváez M, López-Jaramillo P: The hypotensive effect of calcium supplementation during normal pregnancy in Andean women is not related to vascular production of prostacyclin by umbilical arteries. J Obstet Gynecol. 1991;11:93-6.

17. López-Jaramillo P, González MC, Palmer RMJ, Moncada S. The crucial role of physiological $\mathrm{Ca}^{2+}$ concentrations in the production of endothelial nitric oxide and the control of vascular tone. $\mathrm{Br} J$ Pharmacol. 1990;101:489-93.
18. Lopez-Jaramillo P, Narvaez M, Calle A, Rivera J Jacome $P$, Ruano C, et al. Cyclic guanosine $3^{\prime}, 5$ monophosphate concentrations in pre-eclampsia: effects of hydralazine. $\mathrm{Br} \mathrm{J}$ Obstet Gynaecol. 1996;103:33-8.

19. López-Jaramillo P, Terán E, Moncada S. Calcium supplementation prevent pregnancy-induced hypertension by increasing the production of vascular nitric oxide. Med Hypotheses. 1995;45:68-72.

20. Moncada S, Higgs EA, Hodson HF, Knowles RG, López-Jaramillo P, McCall T, Palmer RMJ, Radomski MW, Rees DD, Schultz R: The L-arginine: nitric oxide pathway. J Cardiovas Pharmacol. 1991;17(Suppl 3):S1-S9.

21. López-Jaramillo P. Calcium, nitric oxide, and preeclampsia. Sem Perinatol. 2000;24:33-6.

22. López-Jaramillo P, Casas JP, Serrano NC. Preeclampsia: from epidemiological observations to molecular mechanism. Braz J Med Biol Res. 2001 Oct;34(10):1227-35

23. Serrano NC, Casas JP, Diaz LA, Paez C, Meza CM, Cifuentes R, Monterrosa A, Bautista A, Alarcón MA, Hawe E, Hingorani AD, Vallance P, López-Jaramillo $P$. Endothelial nitric oxide synthase genotype and risk of preeclampsia: a multi-centre case-control study. Hypertension. 2004;44:702-7.

24. Lopez-Jaramillo P, Díaz L A, Pardo A, Parra G, Jaimes $\mathrm{H}$, Chaudhuri $\mathrm{G}$. Estrogen therapy increases plasma concentrations of nitric oxide metabolites in postmenopausal women but increases flow mediated vasodilation only in the younger women. Fertil Steril. 2004;82:1550-5.

25. Var A, Yildirim Y, Onur E, Kuscu NK, Uyanik BS, Goktalay K, et al. Endothelial dysfunction in preeclampsia. Increased homocysteine and decreased nitric oxide levels. Gynecol Obstet Invest. 2003;56:221-4

26. Toth M. Role of tetrahydrobiopterin in the regulation of activity of human placental nitric oxide synthase in normal and pre-eclamptic pregnancies. Orv Hetil. 2002;143:391-8.

27. Cosentino F, Patton S, d'Uscio LV, Werner ER, Werner-Felmayer G, Moreau P, Malinski T, Luscher TF. Tetrahydrobiopterin alters superoxide and nitric oxide release in prehypertensive rats. J Clin Invest. 1998;101:1530-7.

28. Stroes, E, Kastelein J, Cosentino F, Erkelens W, Wever R, Koomans H, Luscher T, Rabelink $T$. Tetrahydrobiopterin restores endothelial function in hypercholesterolemia. J Clin Invest. 1997:99:41-6.

29. Setoguchi S, Mohri M, Shimokawa H, Takeshita A. Tetrahydrobiopterin improves endothelial dysfunction in coronary microcirculation in patients without epicardial coronary artery disease. J Am Coll Cardiol. 2001;38:493-8.

30. Heitzer T, Brockhoff C, Mayer B, Warnholtz A, Mollnau $\mathrm{H}$, Henne S, Meinertz T, Munzel T. Tetrahydrobiopterin improves endotheliumdependen vasodilation in chronic smokers : evidence for a dysfunctional nitric oxide synthase. Circ Res. 2000;86:E36-E41

31. Moncada S, Palmer RM, Higgs EA. Nitric oxide: physiology, pathophysiology, and pharmacology. Pharmacol Rev. 1991;43:109-42.

32. Stuehr DJ. Mammalian nitric oxide synthases Biochem Biophys Acta. 1999;1411:217-30.

33. Moncada S, Higgs A. The L-arginine-nitric oxide pathway. N Engl J Med. 1993;329:2002-12.

34. Nathan $C$, Xie Q-w. Regulation of biosynthesis of nitric oxide. J Biol Chem. 1994;269:13725-8.

35. Kawashima S, Yokoyama M, Dysfunction of endothelial nitric oxide synthase and atherosclerosis. Ar- terioscler Thromb Vasc Biol. 2004;24;998-1005.

36. Garcia RG, Perez M, Maas R, Schwedhelm E, Böger RH, López-Jaramillo P. Plasma concentrations of asymmetric dimethylarginine (ADMA) in metabolic syndrome. Int J Cardiol. 2007;122:176-8.

37. Slaghekke F, Dekker G, Jeffries B. Endogenous inhibitors of nitric oxide and preeclampsia: a review. J Matern Fetal Neonatal Med. 2006;19:447-52.

38. Maas R, Schwedhelm E, Boger RH, Casas JP, López-Jaramillo P, Serrano N, Diaz LA. Plasma concentrations of asymmetrical dimethyl L-arginine (ADMA) in Colombian women with pre-eclampsia. JAMA. 2004:291:823-4.

39. Griendling KK, Sorescu D, Ushio-Fukai M. NAD(P) $\mathrm{H}$ oxidase: role in cardiovascular biology and disease. Circ Res. 2000;86:494-501.

40. Vasquez-Vivar J, Kalyanaraman B, Martasek P, Hogg N, Masters BS, Karoui H, Tordo P, Pritchard KA Jr. Superoxide generation by endothelial nitric oxide synthase: the influence of cofactors. Proc Natl Acad Sci U S A. 1998;95:9220-5.

41. Harma M, Erel O. Measurement of the total antioxidant response in preeclampsia with a novel automated method. Eur J Obstet Gynecol Reprod Biol. 2005;118:47-51.

42. Sagol S, Ozkinay E, Ozsener S. Impaired antioxidant activity in women with pre-eclampsia. Int J Gynaecol Obstet. 1999;64:121-7.

43. Sharma JB, Sharma A, Bahadur A, Vimala N, Satyam A, Mittal S. Oxidative stress markers and antioxidant levels in normal pregnancy and preeclampsia. Int J Gynaecol Obstet. 2006;94:23-7.

44. Reyes LM, Garcia RG, Ruiz SL, Broadhurst D, Aroca G, Davidge ST, López-Jaramillo P. Angiogenic imbalance and plasma lipid alterations in women with preeclampsia from a developing country. Growth Factors. 2012;30:158-66.

45. Aydin S, Benian A, Madazli R, Uludag S, Uzun H, Kaya S. Plasma malondialdehyde, superoxide dismutase, sE-selectin, fibronectin, endothelin-1 and nitric oxide levels in women with preeclampsia. Eur J Obstet Gynecol Reprod Biol. 2004;113:21-5.

46. Takagi Y, Nikaido T, Toki T, Kita N, Kanai M, Ashida $\mathrm{T}$, Ohira S, Konishi I: Levels of oxidative stress and redox-related molecules in the placenta in preeclampsia and fetal growth restriction. Virchows Arch. 2004;444:49-55.

47. Ashok A, Sajal G, Rakesh KS. Role of oxidative stress in female reproduction. Reprod Biol Endocrinol. 2005;3:28

48. Wang Y, Walsh SW. Antioxidant activities and mRNA expression of superoxide dismutase, catalase, and glutathione peroxidase in normal and preeclamptic placentas. J Soc Gynecol Investig. 1996;3:179-84

49. Gulmezoglu AM, Hofmeyr GJ, Oosthuisen MM: Antioxidants in the treatment of severe pre-eclampsia: an explanatory randomized controlled trial. $\mathrm{Br} \mathrm{J}$ Obstet Gynaecol. 1997;104:689-96.

50. Yusuf S, Hawken S, Ounpuu S, Dans T, Avezum A, Lanas $F$, et al. Effect of potentially modifiable risk factors associated with myocardial infarction in 52 countries (the INTERHEART study): case-control study. Lancet. 2004;364:937-52.

51. Caballero B. A nutrition paradox: underweight and obesity in developing countries. N Engl J Med. 2005;352:1514-6.

52. Lopez-Jaramillo P, Pradilla LP, Castillo V, Lahera $\checkmark$. Socioeconomical pathology as determinant of regional differences in the prevalence of metabolic syndrome and pregnancy-induced hypertension. Rev Esp Cardiol. 2007;60:168-78. 
53. López-Jaramillo P, Silva S.Y, Rodriguez Salamanca N, Duran A, Mosquera W, Castillo V. Are nutritioninduced epigenetic changes the link between socioeconomic pathology and cardiovascular diseases? Am J Ther. 2008;15:362-72.

54. Rueda-Clausen C, Silva F, Lopez-Jaramillo P. Epidemic of obesity and overweigh in Latin America and the Caribbean. Int J Cardiol. 2008 Mar 28;125(1):111-2

55. Rueda-Clausen CF, Lahera V, Calderon J, Bolivar IC, Castillo VR, Gutierrez M, Carreno M, Oubina MP, Cachofeiro V, Lopez-Jaramillo P. The presence of abdominal obesity is associated with changes in vascular function independently of other cardiovascular risk factors. Int J Cardiol. 2010;139:32-41.

56. Lopez-Jaramillo P. Cardiometabolic diseases in Latin America: the role of fetal programming in response to maternal malnutrition. Rev Esp Cardiol. 2009;62:670-6.

57. Lopez-Jaramillo P. Defining the research priorities to fight the burden of cardiovascular diseases in Latin America. J Hypertens. 2008;26:1886-9.

58. Seely EW, Solomon CG. Insulin resistance and its potential role in pregnancy-induced hypertension. J Clin Endocrinol Metab. 2003;88:2393-8.

59. Rodie VA, Freeman DJ, Sattar N, Greer I. Preeclampsia and cardiovascular disease: metabolic syndrome of pregnancy? Atherosclerosis. 2004;175:189-202.

60. Kaaja R, Laivuori H, Laakso M, Tikkanen MJ, Ylikorkala O: Evidence of a state of increased insulin resistance in preeclampsia. Metabolism. 1999;48:892-6

61. Sierra-Laguado J, Garcıa RG, Celedón J, ArenasMantilla M, Pradilla LP, Camacho PA, LopezJaramillo $P$. Determination of insulin resistance using the Homeostatic Model Assessment (HOMA) and its relation with the risk of developing pregnancy-induced hypertension. Am J Hypertens. 2007;20:437-42.

62. Lopez-Jaramillo P, Garcia RG, Lopez M. Preventing pregnancy-induced hypertension: are there regional differences for this global problem? J Hypertens. 2005;23:1121-9.

63. Arcaro G, Cretti A, Balzano S, Lechi A, Muggeo M, Bonora E, Bonadonna RC. Insulin causes endothelial dysfunction in humans: sites and mechanisms. Circulation. 2002;105:576-82.

64. Brook RD, Bard RL, Rubenfire M, Ridker PM, Rajagopalan S. Usefulness of visceral obesity (waist/hip ratio) in predicting vascular endothelial function in healthy overweight adults. Am J Cardiol. 2001;88:1264-9.
65. Redman CW, Sacks GP, Sargent IL. Preeclampsia: an excessive maternal inflammatory response to pregnancy. Am J Obstet Gynecol. 1999;180:499-506.

66. Ridker PM, Hennekens CH, Buring JE, Rifai N. C reactive protein and other markers of inflammation in the prediction of cardiovascular disease in women. N Engl J Med. 2000;342:836-43.

67. Roberts JM. Endothelial dysfunction in preeclampsia. Semin Reprod Endocrinol. 1998;16:5-15.

68. Danesh J, Wheeler JG, Hirschfield GM, Eda S, Eiriksdottir G, Rumley A, Lowe GD, Pepys MB, Gudnason V. C-reactive protein and other circulating markers of inflammation in the prediction of coronary heart disease. N Engl J Med. 2004:350:1387-97.

69. Terán E, Escudero C, Moya W, Flores M, Vallance P, López-Jaramillo P. Elevated C-reactive protein and pro-inflammatory cytokines in Andean women with preeclampsia. Int J Gynaecol Obstet. 2001;75:243-9.

70. Garcia RG, Celedón J, Sierra-Laguado J, Alarcon MA, Luengas C, Silva F, Arenas-Mantilla M, Lopez-Jaramillo P. Raised C-reactive protein and impaired flow-mediated vasodilation precede the development of Preeclampsia. Am J Hypertens. 2007;20:98-103.

71. Saikku P, Leinonen M, Mattila K, Ekman MR, Nieminen MS, Mäkelä PH, Huttunen JK, Valtonen V. Serological evidence of an association of a novel Chlamydia, TWAR, with chronic coronary heart disease and acute myocardial infarction. Lancet. 1988 Oct 28;2:983-6.

72. Hu H, Pierce GN, Zhong G. The atherogenic effects of chlamydia are dependent on serum cholesterol and specific to Chlamydia pneumoniae. J Clin Invest. 1999;103:747-53.

73. Kalayoglu MV, Libby P, Byrne GI. Chlamydia pneumoniae as an emerging risk factor in cardiovascular disease. JAMA. 2002;288:2724-31.

74. Heine PR, Ness RB, Roberts JM. Seroprevalence of antibodies to Chlamydia pneumoniae in women with preeclampsia. Obstet Gynecol. 2003;101:221-6.

75. Herrera JA, Chaudhuri G, López-Jaramillo P. Is infection a major risk factor for preeclampsia? Med Hypotheses. 2001;57:393-7.

76. Herrera JA., Parra B, Herrera E, Botero JE, Arced RM, Contreras A, Lopez-Jaramillo P. Periodontal disease severity is related to high levels of Creactive protein in preeclampsia. $\mathrm{J}$ Hypertens. 2007:25:1459-64.
77. Lopez-Jaramillo P, Gomez-Arbelaez D, LopezLopez J. Periodontal disease and hypertension: the pre-eclampsia model in Hispanic population. J Hypertens. 2011;29:1020-1.

78. Bellamy L, Casas JP, Hingorani A, Williams DJ. Pre-eclampsia and risk of cardiovascular disease and cancer in later life: systematic review and meta-analysis. BMJ. 2007;335;974-81.

79. Blaauw J, Pampus VM, Doormaal JJ, Fokkema R, Fidler V, Smit AJ, Aarnoudse JG. Increased intimamedia thickness after early-onset preeclampsia, Clin Obstet Gynecol. 2005;48:489-94.

80. Ness RB, Markovic N, Bass D, Harger G, Roberts JM. Family history of hypertension, heart disease, and stroke among women who develop hypertension in pregnancy. Obstet Gynecol. 2003;102:1366-71.

81. Roberts JM., Taylor RN, Goldfien A. Clinical and biochemical evidence of endothelial cell dysfunction in the pregnancy syndrome preeclampsia. Am J Hypertens. 1991;4:700-8.

82. Reyes L, Garcia R, Ruiz S, Dehghan M, LopezJaramillo P. Nutritional status among women with pre-eclampsia and healthy pregnant and not-pregnant women in Latin American country. J Obstet Gynecol Res. 2012;38:498-504.

83. Ramirez-Velez R, Aguilar de Plata AC, MosqueraEscudero M, Echeverry I, Ortega JG, Salazar B, Rey JJ, Hormiga C, Lopez-Jaramillo P. Influence of regular aerobic exercise on endotheliumdependent vasodilation and cardiorespiratory fitness in pregnant women. J Obstet Gynaecol. 2011;37:1601-8.

Conflictos de intereses: No tenemos conflictos de interés con relación a este manuscrito.

Correspondencia:

Patricio Lopez-Jaramillo MD PhD FACP

Dirección de Investigaciones

Torre Milton Salazar 1 er piso

Calle 155 A No 23-09

Floridablanca, Santander

Colombia

Coreo electrónico:plopezj@gmail.com 